\title{
SIGNATURE JUMPS AND ALEXANDER POLYNOMIALS FOR LINKS
}

\author{
PATRICK M. GILMER AND CHARLES LIVINGSTON \\ (Communicated by Martin Scharlemann)
}

\begin{abstract}
We relate the jumps of the signature function of a link to the roots of its first non-zero higher Alexander polynomial that lie on the unit circle.
\end{abstract}

\section{INTRODUCTION}

A well-known result states that for a knot $K \subset S^{3}$, the absolute value of the classical Murasugi signature, $\sigma_{K}$, is bounded above by the number of roots of the Alexander polynomial, $\Delta_{K}(t)$, on the unit circle, counted with multiplicity. This is most easily proved using Matumoto's result [7] that the signature is the sum of Milnor signatures [8, §5] of the knot at points on the upper half circle. The Milnor signature is the signature of a symmetric bilinear form on a space of dimension given by twice the multiplicity of the root. A similar argument yields a generalization.

Theorem. For any $\omega \in S^{1} \subset \mathbb{C}$, if $\Delta_{K}(\omega) \neq 0$, then the absolute value of the Levine-Tristram signature, $\sigma_{K}(\omega)$, is bounded above by the number of roots (counted with multiplicity) of the Alexander polynomial at points on the unit circle with real parts greater than that of $\omega$.

Generalizing this to links is non-trivial. The Alexander polynomial of a link $L$ can be identically zero, and if non-zero, it can have roots at -1 , which is problematic since $\sigma_{L}=\sigma_{L}(-1)$. Also, following some of the approaches that work in the case of knots leads to subtle technical points that require lengthy arguments to overcome. Our purpose here is to present an approach to a generalization based on simultaneous row and column operations.

\section{Statements of Results}

Let $L$ be an oriented link in $S^{3}$, let $\mu_{L}$ be the number of components of $L$, and let $\Delta_{L}$ denote the Alexander polynomial of $L$. One also has higher Alexander polynomials, $\Delta_{i}(L)$, where $\Delta_{L}=\Delta_{1}(L)$. The polynomial $\Delta_{i}(L)$ can be defined as the greatest common divisor of the set of all $\left(2 g+\mu_{L}-i\right)$-minors of $t V-V^{\top}$, where $V$ is a Seifert matrix for $L$ associated to a connected Seifert surface of genus $g$; these are well defined up to multiplication by $\pm t^{k}$ for some $k$. Let $A_{L} \in \mathbb{Z}\left[t, t^{-1}\right]$ be the

\footnotetext{
Received by the editors September 2, 2015 and, in revised form, December 20, 2015 and January 28, 2016.

2010 Mathematics Subject Classification. Primary 57M25.

Key words and phrases. Link signature.

The first author was partially supported by NSF-DMS-1311911.

The second author was partially supported by a grant from the Simons Foundation and by NSF-DMS-1505586.
} 
first of these higher Alexander polynomials of $L$ which is non-zero. By convention, a 0 -minor is taken to be 1 . Thus every link $L$ has $\Delta_{i}(L)=1$ for some $i$. If $\Delta_{L} \neq 0$, then $A_{L}=\Delta_{L}$.

If $V$ is a Seifert matrix for $L$ coming from a connected Seifert surface, we consider

$$
W(t)=(1-t) V+\left(1-t^{-1}\right) V^{\top} .
$$

Define the Levine-Tristram signature function [4, 11] on $S^{1} \subset \mathbb{C}$ by $\sigma_{L}(\omega)=$ $\operatorname{Sign}(W(\omega))$, where $\omega \in S^{1} \subset \mathbb{C}$. Note that one always has $\sigma_{L}(1)=0$. The Murasugi signature 9 is denoted $\sigma_{L}$ and is defined to be $\sigma_{L}(-1)$. We also consider one-sided limits, $\sigma_{L}^{ \pm}\left(e^{2 \pi i x}\right)=\lim _{y \rightarrow x^{ \pm}} \sigma_{L}\left(e^{2 \pi i y}\right)$, which in turn can be used to define one-sided jumps:

$$
\operatorname{jump}^{ \pm}(\omega)= \pm\left(\sigma_{L}^{ \pm}(\omega)-\sigma_{L}(\omega)\right) .
$$

Thus, jump ${ }^{-}(\omega)$ can be thought of as the jump in signature when arriving at $\omega$ while traveling counterclockwise around the circle. Similarly, jump ${ }^{+}(\omega)$ should be thought of as the jump in signature when departing from $\omega$. We let mult $\omega(f(t))$ denote the multiplicity of $\omega$ as a root of a polynomial $f(t)$.

Theorem 2.1. The signature function $\sigma_{L}(\omega)$ is a step function on $S^{1}$ which can have discontinuities only at roots of $(t-1) A_{L}(t)$. If $\omega \neq \pm 1$, then $\left|\operatorname{jump}^{ \pm}(\omega)\right| \leq$ mult $_{\omega}\left(A_{L}\right)$. In addition, mult $-1\left(A_{L}\right)$ is even, $\mid$ jump $^{ \pm}(-1) \mid \leq(1 / 2)$ mult $_{-1}\left(A_{L}\right)$, and $\mid$ jump $^{ \pm}(1) \mid \leq \mu_{L}-1$.

This easily implies:

Corollary 2.2. The sum $\left|\sigma_{L}\right|+1-\mu_{L}+(1 / 2)$ mult $_{-1}\left(A_{L}\right)$ is less than or equal to the number of roots of $A_{L}$ away from 1 on the unit circle, counted with multiplicity.

We note that if $L$ is a knot, then both $\Delta_{L} \neq 0$ and mult ${ }_{-1}\left(\Delta_{L}\right)=0$. We obtain the following generalization of the result mentioned in the first sentence of this paper.

Theorem 2.3. Assume $\Delta_{L}(t) \neq 0$. The signature function $\sigma_{L}(\omega)$ can have discontinuities only at roots of $\Delta_{L}(t)$. Also $\left|\sigma_{L}\right|+(1 / 2)$ mult $_{-1}\left(\Delta_{L}(t)\right)$ is less than or equal to the number of roots of $\Delta_{L}$ on the unit circle counted with multiplicity.

We also wish to consider the total jump at $\omega: \operatorname{jump}(\omega)=$ jump $^{+}(\omega)+$ jump $^{-}(\omega)$.

Theorem 2.4. If $\omega \neq \pm 1$, jump $(\omega)=2$ mult $_{\omega}\left(A_{L}\right)$ modulo 4. If $\omega \neq \pm 1$ and $\operatorname{mult}_{\omega}\left(A_{L}\right)=1$, then jump ${ }^{+}(\omega)=$ jump $^{-}(\omega)= \pm 1$.

Let $h_{L}$ be the least $i$ such that $\Delta_{i}(L) \neq 0$.

Proposition 2.5. For a link $L$, mult $_{1}\left(A_{L}\right)+\mu_{L}+h_{L}$ is even.

We prove Theorem 2.1 in $\$ 4$ and prove Theorem 2.3 in 95 . We discuss the proof of Theorem 2.4 in $\S 6$. We give a proof of Proposition 2.5 in \$7. In 8 , we discuss further restrictions on the signature function imposed by the structure of the $\mathbb{R}\left[t, t^{-1}\right]$-module presented by the matrix $t V-V^{\top}$. In the last section, we illustrate these results by studying the signature functions of some links. For several of these examples, the signature function jumps at -1 .

Our investigation of the signature function of links began when Stoimenow enquired, in connection with his work on [10, whether $\Delta_{L} \neq 0$ implies that $\left|\sigma_{L}\right|$ is less than or equal to the number of roots of $\Delta_{L}$ on the unit circle, counted with multiplicity. From the published version of [10, we learned of [5], in which an appendix 
by Peter Feller and Livio Liechti affirmatively answers the question that Stoimenow posed. Feller and Liechti's elegant proof ignored the possibility of a discontinuity of the signature function at -1 , but this omission may be easily repaired. We were also influenced by Stavros Garoufalidis' work [2, Lemma 2.1] which discusses some of these results for knots. The method of proof used here is quite different from the methods used by Garoufalidis, Stoimenow and Feller-Liechti. A similar argument to that used in the proof of Lemma 3.1 was sketched by Kate Kearney in [3.

\section{Diagonalizing Hermitian matrices over $\mathbb{R}\left[t, t^{-1}\right]$ LOCAlized AT CERTAIN PRIME IDEALS}

Let $\Lambda=\mathbb{R}\left[t, t^{-1}\right]$ with the involution denoted "bar" determined by $\bar{t}=t^{-1}$ and $\bar{x}=x$ for $x \in \mathbb{R}$. For $\rho \in S^{1} \backslash\{ \pm 1\}$, let $f_{\rho}=t+t^{-1}-\left(\rho+\rho^{-1}\right)$; set $f_{ \pm 1}=t \mp 1$. Note in each case that $f_{\rho}$ is prime in $\Lambda$. For $\rho \in S^{1}$, consider the ring $\Lambda_{\left(f_{\rho}\right)}$, in which all elements prime to $f_{\rho}$ have been inverted. It is a local ring with involution. Also, evaluating $t$ at $\rho$ defines a homomorphism between rings with involution, $\Lambda_{\left(f_{\rho}\right)} \rightarrow \mathbb{C}$, where $\mathbb{C}$ is the complex numbers equipped with complex conjugation as the involution. If $g \in \Lambda_{\left(f_{\rho}\right)}$, this evaluation will be denoted $g(\rho)$, as usual.

A matrix $A$ over $\Lambda_{\left(f_{\rho}\right)}$ presents a $\Lambda_{\left(f_{\rho}\right)}$-module. Two matrices related by an invertible row or column operation present isomorphic modules. The torsion submodule of a finitely generated $\Lambda_{\left(f_{\rho}\right)}$-module has an order which is well defined up to multiplication by units $[8, \S 1]$. This order can be written as $\left(f_{\rho}\right)^{e}$, where $\left(f_{\rho}\right)^{e}$ is the maximal power of $f_{\rho}$ that divides the product of the non-zero entries of a diagonal presentation matrix. We denote this exponent $e_{\rho}(A)$.

A Hermitian matrix $H$ over $\Lambda_{\left(f_{\rho}\right)}$ may be evaluated at $t=\omega$ lying in some open neighborhood of $\rho$ on the unit circle to obtain a Hermitian matrix with entries in $\mathbb{C}$. This is because only a finite number of denominators appear among the entries of $H$. Thus we can consider the signature $\sigma(H(\omega))$ for $\omega$ in this neighborhood and the one-sided jumps at $\rho$ (defined in analogy with the signature jumps of links in $\S 1)$ which we denote by jump ${ }^{ \pm}(H, \rho)$. Moreover $\sigma(H(\rho))$ and jump ${ }^{ \pm}(H, \rho)$ are preserved by an invertible row operation over $\Lambda_{\left(f_{\rho}\right)}$ followed by corresponding conjugate column operation. We refer to such a pair of operations as a simultaneous row and column operation.

Lemma 3.1. Assume $\rho \neq \pm 1$. Every Hermitian matrix $H$ over $\Lambda_{\left(f_{\rho}\right)}$ can be converted to a diagonal matrix by performing a sequence of simultaneous row and column operations. Moreover $\left|\operatorname{jump}^{ \pm}(H, \rho)\right| \leq e_{\rho}(H)$.

Proof. For simplicity of notation in this proof, let $f$ denote $f_{\rho}$.

If all entries of $W$ are divisible by $f$, then factor $f$ out of the matrix and proceed with the simpler matrix $W^{\prime}$ constructed by dividing all entries of $W$ by $f$. Since $f$ is symmetric, $W^{\prime}$ is Hermitian. If $W^{\prime}$ can be diagonalized as above, the same operations will diagonalize $W$.

If some diagonal entry is not divisible by $f$, it can be used to clear out a column and row to reduce the problem to one for a smaller matrix.

If all the diagonal entries are divisible by $f$ but some non-diagonal element is not divisible by $f$, arrange, by permuting the rows and columns, that it is the $(2,1)-$ entry. It can be used to clear the first column (not including the $(1,1)$-entry), and 
the conjugate operations clear the top row. The top left $(2,2)$-block looks like

$$
\left(\begin{array}{cc}
a f^{k} & \bar{b} \\
b & c f^{m}
\end{array}\right)
$$

Notice that $b(\rho) \neq 0$ since $b$ is not divisible by $f$. We will choose an $\alpha$, add $\alpha$ times the second row to the first row and add $\bar{\alpha}$ times the second column to the first column, making the $(1,1)$-entry

$$
a f^{k}+\alpha b+\overline{\alpha b}+\alpha \bar{\alpha} c f^{m} .
$$

We want to choose $\alpha$ so that $\alpha b+\overline{\alpha b}$ evaluated at $\rho$ is non-zero. If $b(\rho)+\overline{b(\rho)} \neq 0$, let $\alpha=1$. If $b(\rho)+\overline{b(\rho)}=0$, then let $\alpha=t$. We claim $\rho b(\rho)+\bar{\rho} \overline{b(\rho)} \neq 0$. Suppose otherwise. Then substituting $\overline{b(\rho)}=-b(\rho)$ yields $\rho b(\rho)-\bar{\rho} b(\rho)=0$, so $\rho-\bar{\rho}=0$. But $\rho$ is not fixed by the involution, and we have reached a contradiction. In this way, we can obtain a diagonal entry not divisible by $f$.

Continuing in this way we obtain a diagonal matrix $\mathbb{D}$. As $\mathbb{D}$ is obtained from $H$ by simultaneous row and column operations, $e_{\rho}(H)=e_{\rho}(\mathbb{D})$ and jump ${ }^{ \pm}(H, \rho)=$ jump $^{ \pm}(\mathbb{D}, \rho)$. Finally it is easy to see that the contribution of a diagonal entry of $\mathbb{D}$ to $\operatorname{jump}^{ \pm}(\mathbb{D}, \rho)$ is \pm 1 if the multiplicity of $\rho$ as a root of this diagonal entry is non-zero. Otherwise the contribution of a diagonal entry is zero. As $e_{\rho}(\mathbb{D})$ is the sum over the diagonal positions of the multiplicity of $\rho$ as a root of these entries, $\mid$ jump $^{ \pm}(\mathbb{D}, \rho) \mid \leq e_{\rho}(\mathbb{D})$. The result follows.

\section{Proof of Theorem 2.1}

Let $V$ be an $(n \times n)$ Seifert matrix for a link. The matrix $t V-V^{\top}$ presents a $\Lambda_{-}^{-}$ module $M$. We can perform independent row and column operations to diagonalize $t V-V^{\top}$, ending with a diagonal matrix with diagonal entries $\left[d_{1}, \ldots, d_{k}, 0, \ldots, 0\right]$, where $d_{i} \neq 0$ and $d_{i}$ divides $d_{i+1}$ for $1 \leq i \leq k-1$. This gives a decomposition:

$$
M=\Lambda^{n-k} \bigoplus_{i=1}^{k} \frac{\Lambda}{\left\langle d_{i}\right\rangle} .
$$

It follows that $A_{L}=\Delta_{n-k+1}(L) \sim_{\Lambda} \prod_{i} d_{i}$; where we let $\sim_{R}$ mean equal up to a multiplicative factor from the units of a ring $R$.

The matrix $W=(1-t) V+(1-t) V^{\top}$ presents a $\Lambda$-module, say, $N$. Since

$$
W=t^{-1}(1-t)\left(t V-V^{\top}\right)
$$

the same row and column operations that diagonalize $t V-V^{\top}$ also diagonalize $W$ and we see that

$$
N=\Lambda^{n-1} \bigoplus_{i=1}^{k} \frac{\Lambda}{\left\langle(1-t) d_{i}\right\rangle} .
$$

4.1. Step function. We first want to observe that, as in the case of knots, the signature function is a step function. There is a diagonalization of $W$ over the field of fractions $\mathbb{R}(t)$. That is, there is a determinant one matrix $A(t)$ with entries in $\mathbb{R}(t)$ such that $A(t) W(t) A^{\top}\left(t^{-1}\right)$ is diagonal, with diagonal entries rational functions: $\left[q_{1}, \ldots, q_{k}, 0, \ldots, 0\right]$. (Since the module $N$ described above becomes an $n-k$ dimensional vector space with $\mathbb{R}(t)$ coefficients, we can use the same value of $k$ here as above.) For all but the finite set of $\rho \in S^{1}$ for which $A(\rho)$ is not defined, this provides a diagonalization of $W(\rho)$. Away from this set of singular values, we see that the signature of $W$ can jump only at zeroes and poles of the diagonal entries. 
Thus, the signature function is a step function; in particular, it has a finite number of discontinuities.

4.2. Jumps away from $\rho= \pm 1$. Let $\rho \in S^{1}$ be a fixed complex number. By Lemma 3.1, we can diagonalize $W$ over the ring $\Lambda_{\left(f_{\rho}\right)}$. Since this matrix presents the module $N \otimes \Lambda_{\left(f_{\rho}\right)}$, we have that after reordering the entries, this diagonalization of $W$ has entries $\left[\alpha_{1}\left(f_{\rho}\right)^{\epsilon_{1}}, \ldots, \alpha_{k}\left(f_{\rho}\right)^{\epsilon_{k}}, 0, \ldots, 0\right]$ where the $\alpha_{i}$ are units in $\Lambda_{\left(f_{\rho}\right)}$ and $\left(f_{\rho}\right)^{\epsilon_{i}}$ is the maximum power of $f_{\rho}$ dividing $d_{i}$.

The jumps at the discontinuities, jump ${ }^{ \pm}$, arise from the diagonal terms for which $\epsilon_{i}>0$. It is now evident that these jumps are bounded by the multiplicity of $\rho$ in $A_{L}$, as was to be proved. Thus $\sigma_{L}(\omega)$ is a step function as claimed.

4.3. Jump at -1 . The diagonalization lemma, Lemma 3.1 does not apply for $\rho=-1$ (see 99.3$)$. A transformation corrects for this. Let $W^{*}$ be the Hermitian matrix $\left(1-t^{2}\right) V+\left(1-t^{-2}\right) V^{\top}$. The jumps of the signature function of $W$ at -1 correspond to the jumps of the signature function of $W^{*}$ at $\rho=\sqrt{-1}$. Notice that $f_{\rho}=t^{-1}+t$.

The diagonalization of $W^{*}$ in $\Lambda_{\left(f_{\rho}\right)}$ has non-zero entries of the following types:

- $\alpha_{i}$, where $\alpha_{i}$ is a unit.

- $\beta_{i}\left(f_{\rho}\right)^{b_{i}}$, where $\beta_{i}(\rho)>0$ and $b_{i}$ is odd.

- $\gamma_{i}\left(f_{\rho}\right)^{c_{i}}$, where $\gamma_{i}(\rho)<0$ and $c_{i}$ is odd.

- $\delta_{i}\left(f_{\rho}\right)^{d_{i}}$, where $\delta_{i}(\rho)>0$ and $d_{i}$ is even.

- $\eta_{i}\left(f_{\rho}\right)^{e_{i}}$, where $\eta_{i}(\rho)<0$ and $e_{i}$ is even.

Suppose that the number of elements for type $\beta, \gamma, \delta$ and $\eta$ are given by $B$, $C, D$, and $E$, respectively. The total jump in the signature function of $W$ at -1 is 0 , by conjugation symmetry, so the same is true for the total jump in the signature function of $W^{*}$ at $\sqrt{-1}$. This implies that $B=C$. We then see that $\mid$ jump $^{ \pm}\left(W^{*}, \sqrt{-1}\right)|\leq| D-E \mid$. Since $D$ and $E$ both correspond to diagonal elements for which $f_{\rho}$ has even exponent, it is now clear that the jump is at most one half the multiplicity of -1 in $A_{L}$. It follows that mult $-1\left(A_{L}\right)$ is even and $\mid$ jump $^{ \pm}(W,-1) \mid \leq$ $(1 / 2)$ mult $_{-1}\left(A_{L}\right)$, as was to be proved.

4.4. Jump at 1 . Since $\sigma_{L}(1)=0, \mid$ jump $^{ \pm}(1)|=| \sigma_{L}^{ \pm}(1) \mid$. We wish to show that $\mu_{L}-1$ is an upper bound for $\left|\sigma_{L}^{ \pm}(1)\right|$. We let $t=\cos (2 \theta)+\sqrt{-1} \sin (2 \theta)$. Expressing $W(t)=(1-t) V+\left(1-t^{-1}\right) V^{\top}$ in terms of $\theta$ and simplifying, we find

$$
W(t)=-\sin (2 \theta)\left(\sqrt{-1}\left(V-V^{\top}\right)-\tan (\theta)\left(V+V^{\top}\right)\right) .
$$

The matrix $V-V^{\top}$ gives the intersection form for the Seifert surface. Thus we may take $V-V^{\top}$ to be the direct sum of $g$ copies of $\left[\begin{array}{cc}0 & 1 \\ -1 & 0\end{array}\right]$ direct sum with a $\left(\mu_{L}-1\right) \times\left(\mu_{L}-1\right)$ zero matrix. It follows that the form $i\left(V-V^{\boldsymbol{\top}}\right)$ has $g$ eigenvalues 1 and $g$ eigenvalues -1 . After a small perturbation, the number of positive and negative eigenvalues will both continue to be at least $g$, so that the absolute value of the signature is at most $\mu_{L}-1$. (Alternatively, the matrix $\sqrt{-1}\left(V-V^{\mathrm{T}}\right)$ is congruent to a diagonal matrix with its first $2 g$ diagonal entries alternating between 1 and -1 . Thus, there are exactly $g$ sign changes in the sequence of the first leading $2 g$ principle minors of this congruent matrix. By the continuity of determinants, the same is true for a small perturbation. It follows that the signature of the upper left $2 g \times 2 g$ block of a small perturbation is also zero. This upper left $2 g \times 2 g$ 
block is non-singular. It follows that the absolute value of the signature of a small perturbation is at most $\mu_{L}-1$.)

\section{Proof of Theorem 2.3}

Lemma 5.1. If $\Delta_{L}(t) \neq 0$, then $\operatorname{mult}_{1}\left(\Delta_{L}(t)\right) \geq \mu_{L}-1$.

Proof. Since $\Delta_{L}(t) \neq 0$, the $\Lambda$-module $M$ presented by $V-t V^{\top}$, has no free summand: $M \cong \bigoplus_{i=1}^{n} \frac{\Lambda}{\left\langle d_{i}\right\rangle}$ where each $d_{i}$ divides $d_{i+1}$ and is non-zero. Tensoring with $\mathbb{R}$, viewed as a $\Lambda$-module with trivial action (formally, $\mathbb{R} \cong \frac{\Lambda}{\langle 1-t\rangle}$ ), yields the $\mathbb{R}$-vector space presented by $V-V^{\top}$. For a link of $\mu$ components, this is isomorphic to $\mathbb{R}^{\mu-1}$ (see the description of $V-V^{\top}$ in $\$ 4.4$ ). However, $\frac{\Lambda}{\left\langle d_{i}\right\rangle} \otimes \frac{\Lambda}{\langle 1-t\rangle}$ is trivial unless $d_{i}$ is divisible by $(1-t)$. Thus, $(\mu-1)$ of the $d_{i}$ are divisible by $(1-t)$. This implies the statement of the lemma.

By Theorem 2.1 $\mu_{L}-1 \geq \mid$ jump $^{ \pm}(1) \mid$. Using the triangle inequality, we easily obtain Theorem 2.3 from Corollary 2.2 .

Remark 5.2. For $L=8 n 8(0,0,0)$ or $8 n 8(1,0,0)$, discussed in 9.2 .2 below, we have $\Delta_{L}(t)=0$, and mult $_{1}\left(A_{L}(t)\right)=2<3=\mu_{L}-1$. Thus Lemma 5.1 cannot be modified to read mult ${ }_{1}\left(A_{L}(t)\right) \geq \mu_{L}-1$ in the situation where $\Delta_{L}(t)=0$. By adapting the argument given above, one can show that

$$
\operatorname{mult}_{1}\left(A_{L}\right) \geq \mu_{L}-h_{L} \text {. }
$$

Note that Proposition 2.5 says that this inequality is also a congruence modulo two.

\section{Proof of Theorem 2.4}

Let $\rho \neq \pm 1$. If $\mathfrak{d}_{i}$ is an entry of the diagonal matrix $\mathbb{D}$ appearing in 4.2 and has mult ${ }_{\rho}\left(\mathfrak{d}_{i}\right)$ odd, then $\mathfrak{d}_{i}$ contributes \pm 2 to $\operatorname{jump}(\rho)$. If $\mathfrak{d}_{i}$ is an entry of $\mathbb{D}$ and has $\operatorname{mult}_{\rho}\left(\mathfrak{d}_{i}\right)$ even, then $\mathfrak{d}_{i}$ contributes 0 to jump $(\rho)$. If mult ${ }_{\rho}\left(A_{L}\right)$ is odd, then an odd number of entries $\left\{\mathfrak{d}_{i}\right\}$ have mult ${ }_{\rho}\left(\mathfrak{d}_{i}\right)$ odd. If mult ${ }_{\rho}\left(A_{L}\right)$ is even, then an even number of entries $\left\{\mathfrak{d}_{i}\right\}$ have mult ${ }_{\rho}\left(\mathfrak{d}_{i}\right)$ odd. The result stated in the first sentence follows.

If $\rho \neq \pm 1$, and $\operatorname{mult}_{\rho}\left(A_{L}\right)=1$, then $\operatorname{mult}_{\rho}\left(\mathfrak{d}_{i}\right)$ is non-zero for exactly one $i$. The result stated in the second sentence follows.

\section{Proof of Proposition 2.5]}

Let $W^{* *}=\left(1-t^{4}\right) V+\left(1-t^{-4}\right) V^{\top}$. The jumps of the signature function of $W$ at 1 correspond to the jumps of the signature function of $W^{* *}$ at $\rho=\sqrt{-1}$. Thus it follows that

$$
\text { jump }^{+}\left(W^{* *}, \sqrt{-1}\right)=- \text { jump }^{-}\left(W^{* *}, \sqrt{-1}\right) .
$$

Notice that $\left(1-t^{4}\right)=(1-t)(1+t)\left(1+t^{2}\right)$ and $f_{\rho}=t^{-1}+t$. Moreover, up to $\sim_{\Lambda}, f(t)=1-t$ is the only irreducible polynomial for which $f\left(t^{4}\right)$ has $1+t^{2}$ as a factor. It follows that $e_{t-1}(W)=e_{t+t^{-1}}\left(W^{* *}\right)$. The argument in 4.3 shows that $e_{t+t^{-1}}\left(W^{* *}\right)$ is even. Thus $e_{t-1}(W)$ is even. But

$$
e_{t-1}(W)=e_{t-1}\left((1-t)\left(t V-V^{\top}\right)\right)=e_{t-1}\left(t V-V^{\top}\right)+2 g+\mu_{L}-h_{L} .
$$

As $\operatorname{mult}_{1}\left(A_{L}\right)=e_{t-1}\left(t V-V^{\top}\right)$, the result follows. 


\section{A MOdUle APPROACH TO THE SIGNATURE FUNCTION}

The proof of Lemma 3.1 and of Theorem 2.1 in the case of $\rho=-1$ (as given in (4.3) point to a general approach to understanding the signature function. Let $V$ be an $n \times n$ Seifert matrix for a link $L$. The $\Lambda$-module $M$ presented by $V-t V^{\top}$ has a direct sum composition

$$
M \cong \frac{\Lambda}{\left\langle d_{1}\right\rangle} \oplus \frac{\Lambda}{\left\langle d_{2}\right\rangle} \cdots \oplus \frac{\Lambda}{\left\langle d_{k}\right\rangle} \oplus \Lambda^{n-k},
$$

where the $d_{i} \in \Lambda$ are non-zero and $d_{i}$ divides $d_{i+1}$ for all $i<k$. We have that $A_{L} \sim_{\Lambda} \prod d_{i}$; also, $\Delta_{L} \neq 0$ if and only if $n=k$. More generally, $h_{L}=n-k+1$. Let $X$ denote the infinite cyclic cover of $S^{3} \backslash L$ specified by the linking number. According to [6. Thm 6.5], the $\mathbb{Z}\left[t, t^{-1}\right]$-module $H_{1}(X, \mathbb{Z})$ is presented by the matrix $V-t V^{\top}$. It follows that $M$ is a description of $H_{1}(X, \mathbb{R})$ as a $\Lambda$-module.

For any $\rho \in S^{1}$, let $\phi_{o}(L, \rho)$ and $\phi_{e}(L, \rho)$ be the number of $d_{i}$ in which $f_{\rho}$ has a positive exponent that is odd or even, respectively. Bounds on the signature function are easily given in terms of these functions; the proofs follow along the same lines as our earlier work. Here is an example of the type of result that can be attained in this way.

Theorem 8.1. If $\rho \neq \pm 1$, then $|\operatorname{jump}(\rho)| \leq 2 \phi_{o}(L, \rho)$ and $|\operatorname{jump}(\rho)|=2 \phi_{o}(L, \rho)$ modulo 4. Also $\left|\operatorname{jump}_{ \pm}(-1)\right| \leq \phi_{e}(L,-1)$ and $\left|\operatorname{jump}_{ \pm}(-1)\right|=\phi_{e}(L,-1)$ modulo 2. Thus

$$
\left|\sigma_{L}-\sigma_{L}^{+}(1)\right| \leq \phi_{e}(L,-1)+\sum_{\rho \in S^{1} \backslash\{-1,1\}} \phi_{o}(L, \rho) .
$$

\section{Some EXAmples}

In this section we will present some examples that illustrate our results. Notice that all the polynomials presented are in $\mathbb{Z}\left[t, t^{-1}\right]$ and are defined up to multiplication by a unit, $\pm t^{i}$. When possible, we normalize polynomials $f$ so that $f \in \mathbb{Z}[t]$ and $f(0) \neq 0$.

9.1. A family of links with zero Alexander polynomial. Consider the family of 3-component links $L_{n}$ illustrated in Figure 1.

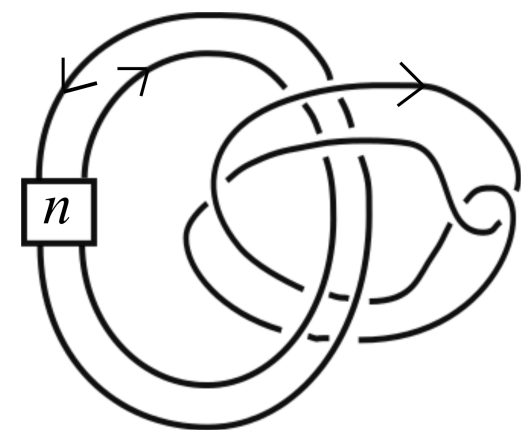

FiguRE 1. Link with $n$ denoting full twists.

There is an obvious disconnected Seifert surface consisting of an annulus and a punctured torus. We tube these together to obtain a connected Seifert surface with 
Seifert matrix

$$
V_{n}=\left(\begin{array}{cccc}
-1 & 1 & 0 & 0 \\
0 & -1 & 1 & 0 \\
0 & 1 & n & 0 \\
0 & 0 & 0 & 0
\end{array}\right)
$$

We have that $\Delta_{L_{n}}=0 ; A_{L_{n}}=\Delta_{2}\left(L_{n}\right)=(t-1)\left((n+1) t^{2}-(n+2) t+(n+1)\right)$;

$$
\sigma_{L_{n}}= \begin{cases}-3 & \text { if } n \leq-2 \\ -1 & \text { if } n \geq-1\end{cases}
$$

Thus $A_{L_{0}}=(t-1)^{3}$ and $\sigma_{L_{0}}=-1$. As $A_{L_{0}}$ has no roots on $S^{1} \backslash\{1\}, \sigma_{L_{0}}$ is identically -1 on $S^{1} \backslash\{1\}$.

Similarly, $A_{L_{-1}}=(t-1)$ and $\sigma\left(L_{-1}\right)=-1$. As $A_{L_{-1}}$ has no roots on $S^{1} \backslash\{1\}$, $\sigma_{L_{-1}}$ is identically -1 on $S^{1} \backslash\{1\}$.

For $n \notin\{0,-1\}, A_{L_{n}}$ has a single conjugate pair of roots $\alpha_{n}$ and $\bar{\alpha}_{n}$ on $S^{1} \backslash\{1\}$ with real part $\Re\left(\alpha_{n}\right)=\frac{n+2}{2(n+1)}$, where $\sigma_{L_{n}}$ must make a total jump of \pm 2 . The one-sided jump at 1 must have absolute value less than or equal to 2 . It follows that if $n \leq-2$, then

$$
\sigma_{L_{n}}\left(e^{\pi i x}\right)= \begin{cases}0 & \text { if } x=0 \\ -1 & \text { if } 0<x<\frac{1}{\pi} \arccos \left(\frac{n+2}{2(n+1)}\right), \\ -2 & \text { if } x=\frac{1}{\pi} \arccos \left(\frac{n+2}{2(n+1)}\right), \\ -3 & \text { if } \frac{1}{\pi} \arccos \left(\frac{n+2}{2(n+1)}\right)<x \leq 1 .\end{cases}
$$

If $n \geq 1$, then

$$
\sigma_{L_{n}}\left(e^{\pi i x}\right)= \begin{cases}0 & \text { if } x=0, \\ 1 & \text { if } 0<x<\frac{1}{\pi} \arccos \left(\frac{n+2}{2(n+1)}\right), \\ 0 & \text { if } x=\frac{1}{\pi} \arccos \left(\frac{n+2}{2(n+1)}\right), \\ -1 & \text { if } \frac{1}{\pi} \arccos \left(\frac{n+2}{2(n+1)}\right)<x \leq 1 .\end{cases}
$$

9.2. Low crossing links with $\Delta_{L}(-1)=0$. In Thistlethwaite's list of links with ten or fewer crossings, there are 16 links for which $\Delta_{L}(-1)=0$; these are $L 8 n 6$, L8n8, L9n18, L9n19, L9n27, L10n32, L10n36, L10n56, L10n57, L10n59, L10n91, L10n93, L10n94, L10n104, L10n107, and L10n111. We will investigate the first three of these links using the Seifert matrices given by LinkInfo [1. In LinkInfo, links are equipped with specified orientations and all orientations, up to simultaneous reversal of all components, are considered. These orientations are described by a zero-one vector of length $\mu-1$.

9.2.1. $L 8 n 6$. We study the $3-$ component link $L 8 n 6$ with its different orientations.

Consider first $L 8 n 6(0,0)$. One computes $\sigma_{L}(-1)=3, A_{L}=\Delta_{L}=(t+1)^{2}(t-1)^{2}$. Thus the signature function can only jump at \pm 1 . It follows that the one-sided jump at 1 can be at most 2 . Also, $\mid$ jump $^{ \pm}(-1) \mid \leq 1$. The only possible signature function consistent with the above is given by

$$
\sigma_{L}\left(e^{\pi i x}\right)= \begin{cases}0 & \text { if } x=0 \\ 2 & \text { if } 0<x<1 \\ 3 & \text { if } x=1\end{cases}
$$


Next, we consider $L 8 n 6(1,0)$. One computes $\sigma_{L}(-1)=-5, A_{L}=\Delta_{L}=$ $(t-1)^{2}(t+1)^{2}\left(t^{2}-t+1\right)$. The last factor is the cyclotomic polynomial with roots the primitive 6 th roots of unity. Thus the signature function can only jump at these 6 th roots of unity and at \pm 1 . The total jump at the 6 th roots of unity must be \pm 2 . The one-sided jump at 1 can be at most 2 . Also, $\mid$ jump ${ }^{ \pm}(-1) \mid \leq 1$. The only possible signature function consistent with the above is given by

$$
\sigma_{L}\left(e^{\pi i x}\right)= \begin{cases}0 & \text { if } x=0 \\ -2 & \text { if } 0<x<\frac{1}{3}, \\ -3 & \text { if } x=\frac{1}{3} \\ -4 & \text { if } \frac{1}{3}<x<1 \\ -5 & \text { if } x=1 .\end{cases}
$$

Finally, consider $L 8 n 6(0,1)$. One computes $\sigma_{L}(-1)=-1, A_{L}=\Delta_{L}=$ $(t-1)^{2}(t+1)^{2}$. Thus the signature function can jump only at \pm 1 . The onesided jump at 1 can be at most 2 . Also, $\mid$ jump $^{ \pm}(-1) \mid \leq 1$. After calculating $\sigma_{L}(\sqrt{-1})=0$, we conclude that

$$
\sigma_{L}\left(e^{\pi i x}\right)= \begin{cases}0 & \text { if } 0 \leq x<1 \\ -1 & \text { if } x=1\end{cases}
$$

The computations and reasoning for $\operatorname{L8n6}(0,1)$ also hold for $\operatorname{L8n6}(1,1)$.

9.2.2. $L 8 n 8$. The link $L 8 n 8(0,0,0)$ has four components. It is built by replacing each component of a Hopf link with a pair of unlinked oppositely oriented components. It has a disconnected Seifert surface composed of two annulli. One can easily work out, by hand, that $\Delta_{L}=0, A_{L}=\Delta_{2}(L)=(t-1)^{2}$, and $\sigma_{L}(\omega)=0$, for all $\omega$.

The link $L 8 n 8(1,0,0)$ is constructed from a Hopf link by replacing one component with a pair of unlinked components oriented in the same way and replacing the other component with a pair of unlinked oppositely oriented components. The computation in this case are most easily carried out with computer assistance. They yield the following: $\Delta_{L}=0, A_{L}=\Delta_{2}(L)=(t-1)^{2}$, and $\sigma_{L}(-1)=0$. One then can conclude that $\sigma_{L}(\omega)=0$, for all $\omega$.

For $L 8 n 8(1,0,1)$, which is obtained by taking a positive Hopf link and replacing each component by a pair of unlinked components oriented in the same way, $A_{L}=$ $\Delta_{L}=(t-1)^{3}(t+1)^{2}, \sigma_{L}(-1)=-4$. The only possible signature function consistent with the above is given by

$$
\sigma_{L}\left(e^{\pi i x}\right)= \begin{cases}0 & \text { if } x=0 \\ -3 & \text { if } 0<x<1 \\ -4 & \text { if } x=1\end{cases}
$$

The other orientations on $L 8 n 8$ are obtained from the above three by symmetries (some reversing the ambient orientation), and so we do not consider these other orientations.

9.2.3. $L 9 n 18$. Consider the 2-component link $L 9 n 18(0)$. One computes from the Seifert matrix that $A_{L}=\Delta_{L}=(t-1)(t+1)^{2}\left(t^{2}-t+1\right)^{2}$ and $\sigma_{L}=6$. The last factor is the square of the cyclotomic polynomial with roots the primitive 6 th roots of unity. Thus the signature function can only jump at these 6 th roots of 
unity and at \pm 1 . The total jump at the 6 th roots of unity are either zero, or \pm 4 . The one-sided jump at 1 can be at most 1 . Also, $\mid$ jump $^{ \pm}(-1) \mid \leq 1$. The only possible signature function consistent with the above is given by

$$
\sigma_{L}\left(e^{\pi i x}\right)= \begin{cases}0 & \text { if } x=0 \\ 1 & \text { if } 0<x<\frac{1}{3} \\ 3 & \text { if } x=\frac{1}{3} \\ 5 & \text { if } \frac{1}{3}<x<1 \\ 6 & \text { if } x=1\end{cases}
$$

Consider $L 9 n 18(1)$. One computes $\sigma_{L}=-2$ and $A_{L}(t)=\Delta_{L}=(t-1)(t+1)^{2}$. Thus the signature function can only jump at \pm 1 . The one-sided jump at 1 can be at most 1 . Also, $\mid$ jump $^{ \pm}(-1) \mid \leq 1$. The signature function consistent with the above is given by

$$
\sigma_{L}\left(e^{\pi i x}\right)= \begin{cases}0 & \text { if } x=0 \\ -1 & \text { if } 0<x<1 \\ -2 & \text { if } x=1\end{cases}
$$

9.3. A 3-component link with $\Delta_{L}=(t-1)^{2}(t+1)^{2}$ and zero signature function. Consider the Seifert matrix

$$
V=\left(\begin{array}{cccc}
1 & -1 & 1 & 1 \\
0 & 0 & 0 & -1 \\
1 & 0 & 0 & 2 \\
1 & -1 & 2 & 0
\end{array}\right)
$$

Pick a 3-component $\operatorname{link} L$ with this Seifert matrix. One computes $\Delta_{L}=$ $(t-1)^{2}(t+1)^{2}$ and $\sigma_{L}(\sqrt{-1})=\sigma_{L}(-1)=0$. Since $\sigma_{L}(\omega)$ can only jump at \pm 1 , we see that $\sigma_{L}(\omega)$ is identically zero.

This example demonstrates that Lemma 3.1 cannot be extended to the case $\rho=-1$. Suppose $W$ can be diagonalized as a Hermitian form over $\Lambda_{(t+1)}$. Then $W$ diagonalized must have all entries self-conjugate. Thus, any entry divisible by $1+t$ must be divisible by an even power of $t+1$. It follows that exactly one entry can be divisible by $1+t$, and this entry must have exponent two. However, this would imply that $\sigma_{L}(\omega)$ has a one-sided jump at -1 , yielding the desired contradiction.

\section{ACKNOWLEDGMEnTs}

The authors wish to thank Alexander Stoimenow for early discussions related to this material. The authors are especially grateful to the referee for comments that significantly improved the paper.

\section{REFERENCES}

[1] J. C. Cha and C. Livingston, LinkInfo: Table of Link Invariants, http://www.indiana.edu/ linkinfo, August 10, 2015.

[2] S. Garoufalidis, Does the Jones polynomial determine the signature of a knot?, arXiv:math/ 03102036.

[3] M. Kate Kearney, The stable concordance genus, New York J. Math. 20 (2014), 973-987. MR.3291607

[4] J. Levine, Invariants of knot cobordism, Invent. Math. 8 (1969), 98-110; addendum, ibid. 8 (1969), 355. MR0253348 
[5] L. Liechti, Signature, positive Hopf plumbing and the Coxeter transformation, Osaka Journal of Mathematics (to appear), arXiv:1401.5336.

[6] W. B. Raymond Lickorish, An introduction to knot theory, Graduate Texts in Mathematics, vol. 175, Springer-Verlag, New York, 1997. MR.1472978

[7] Takao Matumoto, On the signature invariants of a non-singular complex sesquilinear form, J. Math. Soc. Japan 29 (1977), no. 1, 67-71. MR0437456

[8] John W. Milnor, Infinite cyclic coverings, Conference on the Topology of Manifolds (Michigan State Univ., E. Lansing, Mich., 1967), Prindle, Weber \& Schmidt, Boston, Mass., 1968, pp. 115-133. MR0242163

[9] Kunio Murasugi, On a certain numerical invariant of link types, Trans. Amer. Math. Soc. 117 (1965), 387-422. MR.0171275

[10] A. Stoimenow, Application of braiding sequences III: Concordance of positive knots, Internat. J. Math. 26 (2015), no. 7, 1550050, 36, DOI 10.1142/S0129167X15500500. MR3357039

[11] A. G. Tristram, Some cobordism invariants for links, Proc. Cambridge Philos. Soc. 66 (1969), 251-264. MR0248854

Department of Mathematics, Louisiana State University, Baton Rouge, Louisiana 70803

E-mail address: gilmer@math.lsu.edu

$U R L$ : www.math.lsu.edu/ gilmer/

Department of Mathematics, Indiana University, Bloomington, Indiana 47405

E-mail address: livingst@indiana.edu

$U R L$ : mypage.iu.edu/ livingst/ 